University of Texas at El Paso

ScholarWorks@UTEP

$3-2015$

\title{
From 1-D to 2-D Fuzzy: A Proof that Interval-Valued and Complex- Valued Are the Only Distributive Options
}

Christian Servin

El Paso Community College, cservin@gmail.com

Vladik Kreinovich

The University of Texas at El Paso, vladik@utep.edu

Olga Kosheleva

The University of Texas at El Paso, olgak@utep.edu

Follow this and additional works at: https://scholarworks.utep.edu/cs_techrep

Part of the Computer Engineering Commons

Comments:

Technical Report: UTEP-CS-15-21

\section{Recommended Citation}

Servin, Christian; Kreinovich, Vladik; and Kosheleva, Olga, "From 1-D to 2-D Fuzzy: A Proof that IntervalValued and Complex-Valued Are the Only Distributive Options" (2015). Departmental Technical Reports (CS). 902.

https://scholarworks.utep.edu/cs_techrep/902

This Article is brought to you for free and open access by the Computer Science at ScholarWorks@UTEP. It has been accepted for inclusion in Departmental Technical Reports (CS) by an authorized administrator of ScholarWorks@UTEP.For more information, please contact Iweber@utep.edu. 


\section{From 1-D to 2-D Fuzzy: A Proof that Interval-Valued and Complex-Valued Are the Only Distributive Options}

\author{
Christian Servin \\ 1Information Technology Dept. \\ El Paso Community College \\ 919 Hunter, El Paso, Texas 79915, USA \\ Email: cservin@gmail.com
}

\author{
Vladik Kreinovich and Olga Kosheleva \\ University of Texas at El Paso \\ El Paso, Texas 79968, USA \\ Emails:vladik@utep.edu,olgak@utep.edu
}

\begin{abstract}
While the usual 1-D fuzzy logic has many successful applications, in some practical cases, it is desirable to come up with a more subtle way of representing expert uncertainty. A natural idea is to add additional information, i.e., to go from 1-D to 2-D (and multi-D) fuzzy logic. At present, there are two main approaches to 2-D fuzzy logic: interval-valued and complexvalued. At first glance, it may seem that many other options are potentially possible. We show, however, that, under certain reasonable conditions, interval-valued and complex-valued are the only two possible options.
\end{abstract}

\section{INTRODUCTION}

Fuzzy logic: reminder. In the traditional two-valued logic, every statement is either true or false; in the computer these values are represented as, correspondingly 1 and 0 . These two choices cannot capture a typical situation when an expert is not $100 \%$ sure about the validity of a statement. To capture such expert uncertainty, L. Zadeh came up with an idea of fuzzy logic, where for each statement, instead of two possible truth values 0 and 1 , we can have degrees of certainty that can take any values from 0 to 1 [6], [17], [22].

Once we extended the truth values from the original 2valued set $\{0,1\}$ to the whole interval $[0,1]$, we need to also extend the propositional operations of the traditional 2-valued logic to this more general set. From the purely mathematical viewpoint, there are many different ways how we can extend operations from a smaller set (in this case, the set $\{0,1\}$ ) to a larger set (in this case, the interval $[0,1]$ ). Which of these extensions should we choose?

One way to choose such operations is to take into account that operations of 2-valued logic have many intuitive properties, such as commutativity, associativity, distributivity, etc. When we select a proper extension, it is desirable to preserve as many properties of the 2-valued logic as possible.

Need for distributivity. Usually, the "and"- and "or"-operation of fuzzy logic (also known as t-norms and t-conorms) are selected so as to satisfy the properties of commutativity and associativity. This still leaves us with plenty of different choices. It is therefore desirable, among all such operations, to select those that satisfy additional properties.

One of such additional natural properties is distributivity, that $A \&(B \vee C)$ is equivalent to $(A \& B) \vee(A \& C)$.
In terms of an "and"-operation $f_{\&}(a, b)$ and an "or"operation $f_{\vee}(a, b)$, this property means that $f_{\&}\left(a, f_{\vee}(b, c)\right)=$ $f_{\vee}\left(f_{\&}(a, b), f_{\&}(a, c)\right)$.

At first glance, it may sound reasonable to require that this property is satisfied for all possible values of $a, b$, and $c$ - just like we require that the similar commutativity and associativity properties are satisfied for all possible tuples of truth values. However, as we have shown in [19], this "absolute" distributivity implies that $f_{\vee}(a, b)=\max (a, b)$, and it is known that sometimes, the expert's use of "or" is better described by other "or"-operations. To make the operations more adequate, we proposed, in [19], to restrict the above equality to cases when $f_{\vee}(b, c)<1$. We then showed that all corresponding pairs of "and"- and "or"-operations are equivalent to $f_{\&}(a, b)=a \cdot b$ and $f_{\vee}(a, b)=a+b$ (to be more precise, to $\left.f_{\vee}(a, b)=\min (a+b, 1)\right)$.

Need for 2-D extensions. The [0, 1]-based fuzzy logic captures many features of expert uncertainty, but it is well known that in some situations, it is not fully adequate to distinguish between different situations.

For example, if we have no information about a given statement, i.e., if it is equally possible that this statement is true or it is false, then it makes sense to describe this uncertainty by the value 0.5 which is located exactly in the middle between "true" (1) and "false" (0).

On the other hand, if have a lot of information about the given statement $S$, and it so happens that we have exactly as many arguments and facts supporting this statement as we have in support of its negation, then it also makes sense to describe this uncertainty by the midpoint value 0.5 .

In both situations, the truth value is the same, but the uncertainty is different. For example, if we add one additional argument in support of the statement, then:

- $\quad$ in the first case, we now have and argument supporting $S$ and no arguments supporting $\neg S$, so the truth value of $S$ should drastically increase, while

- in the second case, the truth value should not change much - since the numbers of statement supporting $S$ and $\neg S$ remains almost equal. 
To distinguish between such situations, it is desirable to supplement the $[0,1]$-valued degree of belief with an additional number (or numbers). The simplest case if when we use one additional number, i.e., when we use two numbers to describe our degree of certainty in a given statement.

Known 2-D distributive extensions of 1-D fuzzy logic. Two 2-D extensions of fuzzy logic are known. The most widely used in interval-valued fuzzy logic, where our degree of certainty in a statement is described by an interval $[\underline{d}, \bar{d}] \subseteq[0,1]$ [12], [13], [16]. This enables us to clearly distinguish between the above two situations:

- $\quad$ the case of complete uncertainty is naturally described by the interval $[0,1]$, while

- the case when have many argument in favor of the statement $S$ and as many arguments against $S$ is naturally described by a degenerate interval $[0,5,0.5]$ consisting of a single number 0.5 .

In principle, we can extend different t-norms and t-conorms to the interval-valued case, by using a natural extension [4], [14]

$$
\begin{gathered}
f([\underline{a}, \bar{a}],[\underline{b}, \bar{b}]) \stackrel{\text { def }}{=}\{f(a, b): a \in[\underline{a}, \bar{a}] \text { and } b \in[\underline{b}, \bar{b}]\}= \\
{[f(\underline{a}, \underline{b}), f(\bar{a}, \bar{b})] .}
\end{gathered}
$$

In particular, if we extend the distributive operations $a \cdot b$ and $a+b$ to

$$
[\underline{a}, \bar{a}] \cdot[\underline{b}, \bar{b}]=[\underline{a} \cdot \underline{b}, \bar{a} \cdot \bar{b}]
$$

and

$$
[\underline{a}, \bar{a}]+[\underline{b}, \bar{b}]=[\underline{a}+\underline{b}, \bar{a}+\bar{b}],
$$

we get a distributive interval-valued logic.

Another useful 2-D distributive extension of the usual fuzzy logic is the complex-valued fuzzy logic, in which the degrees of belief can take any complex values $a+b \cdot \mathrm{i}$, with $\mathrm{i} \stackrel{\text { def }}{=} \sqrt{-1}$; see, e.g., [1], [2], [3], [8], [9], [15]. While it is empirically successful, it is not as widely used an interval-valued fuzzy logic, since it lacks a clear justification and clear interpretation.

Are there other extension? At first glance, it looks like the above two extensions have been rather arbitrarily chosen, and in principle, there are many other extensions. In this paper, we show, however, that interval-valued and complex-valued are the only possible 2-D distributive extensions of the usual fuzzy logic.

In our opinion, this result elevates complex-valued fuzzy logic from the status of one of the mathematically possible extensions to a much higher status of one of the two possible extensions - and will, hopefully lead to a more frequent use of complex-valued fuzzy logic.

\section{Analysis of the Problem AND the Main Result}

Toward precise description of a general 2-D distributive extensions: set of possible values. We start with real-valued operations $\cdot$ and + . We want to extend these operations to a 2-D set extending the set of usual real numbers to a larger set containing an additional element $x$.
In the case of intervals, $x$ is one of the non-degenerate intervals $[\underline{a}, \bar{a}]$, with $\underline{a}<\bar{a}$. In the case of complex numbers, $x$ is either the square root of -1 , or, more generally, any nonreal complex number $a+b \cdot \mathrm{i}$, with $b \neq 0$. In general, $x$ can be anything.

On this extended set, we want to allow multiplication. Thus, we need to consider elements of the type $b \cdot x$ for arbitrary real numbers $b$. We also want to allow addition between real numbers $a$ and the products $b \cdot x$. So, we need to consider elements of the type

$$
a+b \cdot x .
$$

The set of all such elements depends on two parameters $a$ and $b$ and is, therefore, already 2-dimensional. Since we are interested in 2-D extensions, this means that the desired extension cannot contain any other elements. So, each extension is the set of all the elements of type (1).

Addition ("or"-operation) on the set of possible values. Commutativity, associativity, and distributivity help us define the sum of elements of type (1). Indeed, if we have the elements $a+b \cdot x$ and $a^{\prime}+b^{\prime} \cdot x$, then, due to commutativity and associativity of addition, we get

$$
(a+b \cdot x)+\left(a^{\prime}+b^{\prime} \cdot x\right)=\left(a+a^{\prime}\right)+\left(b \cdot x+b^{\prime} \cdot x\right) .
$$

Now, distributivity implies that

$$
b \cdot x+b^{\prime} \cdot x=\left(b+b^{\prime}\right) \cdot x .
$$

Substituting the expression (3) into the formula (2), we get the following formula:

$$
(a+b \cdot x)+\left(a^{\prime}+b^{\prime} \cdot x\right)=\left(a+a^{\prime}\right)+\left(b+b^{\prime}\right) \cdot x .
$$

In other words, we have a component-wise addition.

Comment. It should be mentioned that both for intervals and for complex numbers, we do have component-wise addition.

\section{Multiplication ("or"-operation) on the set of possible val-} ues. Due to distributivity, we have

$$
\begin{gathered}
(a+b \cdot x) \cdot\left(a^{\prime}+b^{\prime} \cdot x\right)= \\
a \cdot b+\left(a \cdot b^{\prime}+a^{\prime} \cdot b\right) \cdot x+\left(b \cdot b^{\prime}\right) \cdot(x \cdot x) .
\end{gathered}
$$

Thus, to describe the product of the new objects, it is sufficient to know the value of $x \cdot x$.

Since all the new elements have the form (1), we thus have

$$
x \cdot x=p+q \cdot x
$$

for some real numbers $p$ and $q$. Let us show that we can simplify this formula by re-selecting the element $x$.

First, instead of the original element $x$, we can select a new element $x^{\prime}=x-\frac{q}{2}$. This new selection does not change the class of elements $a+b \cdot x$ : indeed, we have $x=x^{\prime}+\frac{q}{2}$, and thus, each element $a+b \cdot x$ can be represented as

$$
a+b \cdot\left(x^{\prime}+\frac{q}{2}\right)=\left(a+b \cdot \frac{q}{2}\right)+b \cdot x^{\prime},
$$

i.e., as $a^{\prime}+b \cdot x^{\prime}$, where we denoted

$$
a^{\prime} \stackrel{\text { def }}{=} a+b \cdot \frac{q}{2} \text {. }
$$


For the new element $x^{\prime}=x-\frac{q}{2}$, we have

$$
\begin{gathered}
x^{\prime} \cdot x^{\prime}=\left(x-\frac{q}{2}\right) \cdot\left(x-\frac{q}{2}\right)=x \cdot x-2 \cdot \frac{q}{2} \cdot x+\frac{q^{2}}{4}= \\
x \cdot x-q \cdot x+\frac{q^{2}}{4} .
\end{gathered}
$$

Substituting the formula (6) into this expression, we conclude that

$$
x^{\prime} \cdot x^{\prime}=p+q \cdot x-q \cdot x+\frac{q^{2}}{4},
$$

i.e., that

$$
x^{\prime} \cdot x^{\prime}=p^{\prime}
$$

where we denoted

$$
p^{\prime} \stackrel{\text { def }}{=} p+\frac{q^{2}}{4} .
$$

Thus, without losing generality, we can assume that

$$
x \cdot x=p
$$

for some real number $p$.

There are three possible cases of $p$ : it can be positive, it can be negative, and it can be equal to 0 . Let us consider these three cases one by one.

Case when $p>0$. In this case, we can simplify the formula (8) even more, by considering an alternative element $x^{\prime \prime}=\frac{x}{\sqrt{p}}$. A switch to this element does not change the class of elements $a+b \cdot x$ : indeed, since $x=\sqrt{p} \cdot x^{\prime \prime}$, each element $a+b \cdot x$ has the form

$$
a+b \cdot x=a+b \cdot \sqrt{p} \cdot x^{\prime \prime},
$$

i.e., the form $a+b^{\prime \prime} \cdot x^{\prime \prime}$, where we denoted $b^{\prime \prime} \stackrel{\text { def }}{=} b \cdot \sqrt{p}$.

For the new element $x^{\prime}$, we have

$$
x^{\prime \prime} \cdot x^{\prime \prime}=\frac{x}{\sqrt{p}} \cdot \frac{x}{\sqrt{p}}=\frac{x \cdot x}{p} .
$$

Substituting the expression (8) into this formula, we conclude that

$$
x^{\prime \prime} \cdot x^{\prime \prime}=\frac{p}{p}=1
$$

Thus, without losing generality, in the case of $p>0$, we can conclude that

$$
x \cdot x=1 .
$$

In this case, the general product formula (5) takes the form

$$
(a+b \cdot x) \cdot\left(a^{\prime}+b^{\prime} \cdot x\right)=a^{\prime \prime}+b^{\prime \prime} \cdot x,
$$

where

$$
a^{\prime \prime}=a \cdot a^{\prime}+b \cdot b^{\prime}
$$

and

$$
b^{\prime \prime}=a \cdot b^{\prime}+a^{\prime} \cdot b .
$$

Let us show that if we interpret each element $a+b \cdot x$ as an interval $[a-b, a+b]$, then, for intervals consisting of nonnegative numbers:

- the formula (4) becomes a formula for adding intervals, and
- the formulas (10)-(12) becomes the formulas for multiplying intervals.

Let us consider these two operations one by one.

Case of $p=1$ : addition. If we add two intervals $[\underline{a}, \bar{a}]=$ $[a-b, a+b]$ and $\left[\underline{a}^{\prime}, \overline{a^{\prime}}\right]=\left[a^{\prime}-b^{\prime}, a^{\prime}+b^{\prime}\right]$, then, as we have mentioned earlier, we get an interval

$$
\begin{gathered}
{[\underline{a}+\underline{b}, \bar{a}+\bar{b}]=\left[(a-b)+\left(a^{\prime}-b^{\prime}\right),(a+b)+\left(a^{\prime}+b^{\prime}\right)\right]=} \\
{\left[\underline{a}^{\prime \prime}, \overline{a^{\prime \prime}}\right],}
\end{gathered}
$$

where

$$
\underline{a}^{\prime \prime}=(a-b)+\left(a^{\prime}-b^{\prime}\right)
$$

and

$$
\overline{a^{\prime \prime}}=(a+b)+\left(a^{\prime}+b^{\prime}\right) .
$$

We want to interpret this interval as $\left[a^{\prime \prime}-b^{\prime \prime}, a^{\prime \prime}+b^{\prime \prime}\right]$ for some values $a^{\prime \prime}$ and $b^{\prime \prime}$. For that, we need to select $a^{\prime \prime}$ and $b^{\prime \prime}$ from the conditions that

$$
a^{\prime \prime}-b^{\prime \prime}=(a-b)+\left(a^{\prime}-b^{\prime}\right)
$$

and

$$
a^{\prime \prime}+b^{\prime \prime}=(a+b)+\left(a^{\prime}+b^{\prime}\right) .
$$

Adding equations (13) and (14) and dividing the result by 2 , we conclude that

$$
\begin{gathered}
a^{\prime \prime}=\frac{1}{2} \cdot\left((a-b)+\left(a^{\prime}-b^{\prime}\right)+(a+b)+\left(a^{\prime}+b^{\prime}\right)\right)= \\
\frac{1}{2} \cdot\left(a-b+a^{\prime}-b^{\prime}+a+b+a^{\prime}+b^{\prime}\right)=\frac{1}{2} \cdot(2 a+2 b)= \\
a+b .
\end{gathered}
$$

Similarly, subtracting the equation (14) from the equation (13) and dividing the result by 2 , we conclude that

$$
\begin{gathered}
b^{\prime \prime}=\frac{1}{2} \cdot\left((a+b)+\left(a^{\prime}+b^{\prime}\right)-(a-b)-\left(a^{\prime}-b^{\prime}\right)\right)= \\
\frac{1}{2} \cdot\left(a+b+a^{\prime}+b^{\prime}-a+b-a^{\prime}+b^{\prime}\right)=\frac{1}{2} \cdot\left(2 a^{\prime}+2 b^{\prime}\right)= \\
a^{\prime}+b^{\prime} .
\end{gathered}
$$

Thus, for every two intervals

$$
[a-b, a+b] \text { and }\left[a^{\prime}-b^{\prime}, a^{\prime}+b^{\prime}\right],
$$

their sum is the interval $\left[a^{\prime \prime}-b^{\prime \prime}, a^{\prime \prime}+b^{\prime \prime}\right]$, where $a^{\prime \prime}=a+a^{\prime}$ and $b^{\prime \prime}=b+b^{\prime}$. This is exactly what the formula (4) implies if we interpret each number $a+b \cdot x$ as an interval $[a-b, a+b]$.

Case of $p=1$ : multiplication. If we add multiply intervals $[\underline{a}, \bar{a}]=[a-b, a+b]$ and $\left[\underline{a}^{\prime}, \overline{a^{\prime}}\right]=\left[a^{\prime}-b^{\prime}, a^{\prime}+b^{\prime}\right]$, then, as we have mentioned earlier, we get an interval

$$
\begin{gathered}
{[\underline{a} \cdot \underline{b}, \bar{a} \cdot \bar{b}]=\left[(a-b) \cdot\left(a^{\prime}-b^{\prime}\right),(a+b) \cdot\left(a^{\prime}+b^{\prime}\right)\right]=} \\
{\left[\underline{a}^{\prime \prime}, \overline{a^{\prime \prime}}\right],}
\end{gathered}
$$

where

$$
\underline{a}^{\prime \prime}=(a-b) \cdot\left(a^{\prime}-b^{\prime}\right)
$$


and

$$
\overline{a^{\prime \prime}}=(a+b) \cdot\left(a^{\prime}+b^{\prime}\right) .
$$

We want to interpret this interval as $\left[a^{\prime \prime}-b^{\prime \prime}, a^{\prime \prime}+b^{\prime \prime}\right]$ for some values $a^{\prime \prime}$ and $b^{\prime \prime}$. For that, we need to select $a^{\prime \prime}$ and $b^{\prime \prime}$ from the conditions that

$$
a^{\prime \prime}-b^{\prime \prime}=(a-b) \cdot\left(a^{\prime}-b^{\prime}\right)
$$

and

$$
a^{\prime \prime}+b^{\prime \prime}=(a+b) \cdot\left(a^{\prime}+b^{\prime}\right) .
$$

Adding equations (15) and (16) and dividing the result by 2 , we conclude that

$$
\begin{gathered}
a^{\prime \prime}=\frac{1}{2} \cdot\left((a-b) \cdot\left(a^{\prime}-b^{\prime}\right)+(a+b) \cdot\left(a^{\prime}+b^{\prime}\right)\right)= \\
\frac{1}{2} \cdot\left(a \cdot a^{\prime}-a \cdot b^{\prime}-b \cdot a^{\prime}+b \cdot b^{\prime}+a \cdot a^{\prime}+a \cdot b^{\prime}+b \cdot a^{\prime}+b \cdot b^{\prime}\right)= \\
\frac{1}{2} \cdot\left(2 a \cdot a^{\prime}+2 b \cdot b^{\prime}\right)=a \cdot a^{\prime}+b \cdot b^{\prime} .
\end{gathered}
$$

Similarly, subtracting the equation (16) from the equation (15) and dividing the result by 2 , we conclude that

$$
\begin{gathered}
b^{\prime \prime}=\frac{1}{2} \cdot\left((a+b) \cdot\left(a^{\prime}+b^{\prime}\right)+(a-b) \cdot\left(a^{\prime}-b^{\prime}\right)\right)= \\
\frac{1}{2} \cdot\left(a \cdot a^{\prime}+a \cdot b^{\prime}+b \cdot a^{\prime}+b \cdot b^{\prime}-a \cdot a^{\prime}+a \cdot b^{\prime}+b \cdot a^{\prime}-b \cdot b^{\prime}\right)= \\
\frac{1}{2} \cdot\left(2 a \cdot b^{\prime}+2 a^{\prime} \cdot b\right)=a \cdot b^{\prime}+a^{\prime} \cdot b .
\end{gathered}
$$

Thus, for every two intervals

$$
[a-b, a+b] \text { and }\left[a^{\prime}-b^{\prime}, a^{\prime}+b^{\prime}\right],
$$

their product is the interval $\left[a^{\prime \prime}-b^{\prime \prime}, a^{\prime \prime}+b^{\prime \prime}\right]$, where $a^{\prime \prime}=$ $a \cdot a^{\prime}+b \cdot b^{\prime}$ and $b^{\prime \prime}=a \cdot b^{\prime}+a^{\prime} \cdot b$. This is exactly what the formula (5) with $x \cdot x=1$ implies if we interpret each number $a+b \cdot x$ as an interval $[a-b, a+b]$.

Case when $p>0$ : conclusion. In this case, we get intervalvalued fuzzy logic.

Comment. Main formulas for this derivation can be traced to a pioneering interval paper [20].

Case when $p<0$. In this case, we can simplify the formula (8) by considering an alternative element $x^{\prime \prime}=\frac{x}{\sqrt{|p|}}$. A switch to this element does not change the class of elements $a+b \cdot x$ : indeed, since $x=\sqrt{|p|} \cdot x^{\prime \prime}$, each element $a+b \cdot x$ has the form

$$
a+b \cdot x=a+b \cdot \sqrt{|p|} \cdot x^{\prime \prime},
$$

i.e., the form $a+b^{\prime \prime} \cdot x^{\prime \prime}$, where we denoted $b^{\prime \prime} \stackrel{\text { def }}{=} b \cdot \sqrt{|p|}$.

For the new element $x^{\prime}$, we have

$$
x^{\prime \prime} \cdot x^{\prime \prime}=\frac{x}{\sqrt{|p|}} \cdot \frac{x}{\sqrt{|p|}}=\frac{x \cdot x}{|p|} .
$$

Substituting the expression (8) into this formula, we conclude that

$$
x^{\prime \prime} \cdot x^{\prime \prime}=\frac{p}{|p|}=-1 \text {. }
$$

Thus, without losing generality, in the case of $p<0$, we can conclude that

$$
x \cdot x=-1 .
$$

In this case, the formula (5) takes the form

$$
\begin{gathered}
(a+b \cdot x) \cdot\left(a^{\prime}+b^{\prime} \cdot x\right)= \\
\left(a \cdot b-a^{\prime} \cdot b^{\prime}\right)+\left(a \cdot b^{\prime}+a^{\prime} \cdot b\right) \cdot x,
\end{gathered}
$$

i.e., the usual multiplication formula for complex numbers. Since the formula (2) is also the usual formula for adding complex numbers, we thus get complex-valued fuzzy logic.

Case when $p<0$ : conclusion. In this case, we get complexvalued fuzzy logic.

Case when $p=0$. In this case, the formula (5) implies that

$$
(a+b \cdot x) \cdot\left(a^{\prime}+b^{\prime} \cdot x\right)=a \cdot a^{\prime}+\left(a \cdot b^{\prime}+a^{\prime} \cdot b\right) \cdot x .
$$

This formula is used in linearization approach to uncertainty [10], [11], [18]. In this approach, we start with measurement results $\widetilde{x}_{i}$ which are reasonably accurate, i.e., close enough to actual (unknown) values $x_{i}$ of the corresponding quantities so that we can safely ignore the terms that are quadratic (or higher order) in terms of the measurement error $\Delta x_{i} \stackrel{\text { def }}{=} \widetilde{x}_{i}-x_{i}$.

In general, we are interested in the values of the quantities $y$ which depend on the directly measured quantities $\left.x_{1}, \ldots, x_{n}\right)$ in a known way: $y=f\left(x_{1}, \ldots, x_{n}\right)$. For each such quantity, we are interested in the value $y=f\left(x_{1}, \ldots, x_{n}\right)$ corresponding to the actual values $x_{i}$. In practice, we only have the measurement results $\widetilde{x}_{i}$, so we estimate $y$ as $\widetilde{y}=f\left(\widetilde{x}_{1}, \ldots, \widetilde{x}_{n}\right)$.

How accurate is this estimate? What can we conclude about the actual value $y$ based on this estimate? By definition of the measurement error $\Delta x_{i}$, we have $x_{i}=\widetilde{x}_{i}-\Delta x_{i}$, so we have

$$
f\left(x_{1}, \ldots, x_{n}\right)=f\left(\widetilde{x}_{1}-\Delta x_{1}, \ldots, \widetilde{x}_{n}-\Delta x_{n}\right) .
$$

Since the measurement errors $\Delta x_{i}$ are small, we can expand the expression $f\left(\widetilde{x}_{1}-\Delta x_{1}, \ldots, \widetilde{x}_{n}-\Delta x_{n}\right)$ in Taylor series in terms of $\Delta x_{i}$ and ignore terms which are quadratic (or of higher order) in terms of $\Delta x_{i}$. As a result, we get

$$
y=f\left(x_{1}, \ldots, x_{n}\right)=\widetilde{y}+\sum_{i=1}^{n} y_{i} \cdot \Delta x_{i},
$$

where we denoted $y_{i} \stackrel{\text { def }}{=}-\frac{\partial f}{\partial x_{i}}$.

In particular, if we know the upper bounds $\Delta_{i}$ on the (absolute values of) measurement errors $\Delta x_{i}$ - i.e., if we known that the actual value $x_{i}$ is located in the interval $\left[\widetilde{x}_{i}-\Delta_{i}, \widetilde{x}_{i}+\Delta_{i}\right]-$ then we can conclude that the actual value $y$ is contained in the interval $[\widetilde{y}-\Delta, \widetilde{y}+\Delta]$, where $\Delta \stackrel{\text { def }}{=} \sum_{i=1}^{n}\left|y_{i}\right| \cdot \Delta_{i}$.

For each quantity $y$, the actual value can be represented as

$$
y=\widetilde{y}+\sum_{i=1}^{n} y_{i} \cdot \Delta_{i} .
$$


If we have a second quantity

$$
y^{\prime}=\widetilde{y^{\prime}}+\sum_{i=1}^{n} y_{i}^{\prime} \cdot \Delta_{i},
$$

then their sum is represented as

$$
y+y^{\prime}=\left(\widetilde{y}+\widetilde{y^{\prime}}\right)+\sum_{i}\left(y_{i}+y_{i}^{\prime}\right) \cdot \Delta x_{i} .
$$

Since the quadratic terms can be ignored, for the product, we have a representation

$$
y \cdot y^{\prime}=\left(\widetilde{y} \cdot \widetilde{y^{\prime}}\right)+\sum_{i}\left(\widetilde{y} \cdot y_{i}^{\prime}+\widetilde{y^{\prime}} \cdot y_{i}\right) \cdot \Delta x_{i} .
$$

In particular, for $n=1$, we have general representation as $y=\widetilde{y}+y_{1} \cdot \Delta x_{1}$, and the formulas for the sum and product take the form

$$
y+y^{\prime}=\left(\widetilde{y}+\widetilde{y^{\prime}}\right)+\left(y_{1}+y_{1}^{\prime}\right) \cdot \Delta x_{1}
$$

and

$$
y \cdot y^{\prime}=\left(\widetilde{y} \cdot \tilde{y^{\prime}}\right)+\left(\widetilde{y} \cdot y_{1}^{\prime}+\widetilde{y^{\prime}} \cdot y_{1}\right) \cdot \Delta x_{1} .
$$

For $x=\Delta x_{1}$, these are exactly the formulas (4) and (5) corresponding to $p=0$. Thus, we arrive at the following conclusion.

Case when $p=0$ : conclusion. This case corresponds to a special case of interval-valued fuzzy logic, when intervals are narrow so that we can ignore terms which are quadratic in terms of their width.

General conclusion. We have thus proven that there are only two types of distributive 2-D fuzzy logic: interval-valued or complex-valued.

Comment. From the mathematical viewpoint, the main formulas behind the above result are available in [5], [7], [21].

\section{ACKNOWLEDGMENTS}

This work was supported in part by the National Science Foundation grants HRD-0734825 and HRD-1242122 (CyberShARE Center of Excellence) and DUE-0926721.

\section{REFERENCES}

[1] S. Aghakhani and S. Dick, "An on-line learning algorithm for complex fuzzy logic", Proceedings of the 2010 International IEEE Conference on Fuzzy Sets and Systems FUZZ-IEEE'2010, pp. 1-7.

[2] Z. Chen, S. Aghakhani, J. Man, and S. Dick, "ANCFIS: A Neurofuzzy Architecture Employing Complex Fuzzy Sets", IEEE Transactions on Fuzzy Systems, 2011, Vol. 19, No. 2, pp. 305-322.

[3] S. Dick, "Towards complex fuzzy logic", IEEE Transactions on Fuzzy Systems, 2005, Vol. 13, No. 3, pp. 405-414.

[4] L. Jaulin, M. Kieffer, O. Didrit, and E. Walter, Applied Interval Analysis, with Examples in Parameter and State Estimation, Robust Control and Robotics, Springer-Verlag, London, 2001.
[5] I. L. Kantor and A. S. Solodovnikov, Hypercomplex Numbers: An Elementary Introduction to Algebras, Springer-Verlag, Berlin, New York, 1989.

[6] G. Klir and B. Yuan, Fuzzy Sets and Fuzzy Logic, Prentice Hall, Upper Saddle River, New Jersey, 1995.

[7] M. Koecher and R. Remmert, "The Isomorphism Theorems of Frobenius, Hopf, and Gelfand-Mazur", In: H.-D. Ebbinghaus, H. Hermes, F. Hirzebruch, M. Koecher, K. Mainzer, J. Neukirch, A. Prestel, and R. Remmert, Numbers, Springer Verlag, New York, 1991, pp. 221-248.

[8] O. Kosheleva and V. Kreinovich, "Approximate nature of traditional fuzzy methodology naturally leads to complex-valued fuzzy degrees", Proceedings of the IEEE World Congress on Computational Intelligence WCCI'2014, Beijing, China, July 6-11, 2014.

[9] O. Kosheleva, V. Kreinovich, and T. Ngamsantivong, "Why complexvalued fuzzy? Why complex values in general? A computational explanation", Proceedings of the Joint World Congress of the International Fuzzy Systems Association and Annual Conference of the North American Fuzzy Information Processing Society IFSA/NAFIPS2013, Edmonton, Canada, June 24-28, 2013, pp. 1233-1236.

[10] V. Kreinovich, "Interval computations and interval-related statistical techniques: tools for estimating uncertainty of the results of data processing and indirect measurements", In: F. Pavese and A. B. Forbes (eds.), Data Modeling for Metrology and Testing in Measurement Science, Birkhauser-Springer, Boston, 2009, pp. 117-145.

[11] V. Kreinovich, "Interval computations and interval-related statistical techniques: estimating uncertainty of the results of data processing and indirect measurements", In: F. Pavese (ed.), Advanced Mathematical and Computational Tools in Metrology and Testing AMTCM'X, World Scientific, Singapore, 2015, pp. 38-49.

[12] J. M. Mendel, Uncertain Rule-Based Fuzzy Logic Systems: Introduction and New Directions, Prentice-Hall, Upper Saddle River, New Jersey, 2001.

[13] J. M. Mendel and D. Wu, Perceptual Computing: Aiding People in Making Subjective Judgments, IEEE Press and Wiley, Piscataway, New Jersey, 2010.

[14] R. E. Moore, R. B. Kearfott, and M. J. Cloud, Introduction to Interval Analysis, SIAM Press, Philadelphia, Pennsylviania, 2009.

[15] H. T. Nguyen, V. Kreinovich, and V. Shekhter, "On the possibility of using complex values in fuzzy logic for representing inconsistencies", International Journal of Intelligent Systems, 1998, Vol. 13, No. 8, pp. 683-714.

[16] H. T. Nguyen, V. Kreinovich, and Q. Zuo, "Interval-valued degrees of belief: applications of interval computations to expert systems and intelligent control", International Journal of Uncertainty, Fuzziness, and Knowledge-Based Systems (IJUFKS), 1997, Vol. 5, No. 3, pp. 317-358.

[17] H. T. Nguyen and E. A. Walker, A First Course in Fuzzy Logic, Chapman and Hall/CRC, Boca Raton, Florida, 2006.

[18] S. G. Rabinovich, Measurement Errors and Uncertainty: Theory and Practice, Springer Verlag, Berlin, 2005.

[19] C. Servin and V. Kreinovich, "Towards efficient algorithms for approximating a fuzzy relation by fuzzy rules: case when 'and'- and 'or'-operation are distributive", Proceedings of the 2014 Annual Conference of the North American Fuzzy Information Processing Society NAFIPS'2014, Boston, Massachusetts, June 24-26, 2014.

[20] M. Warmus, "Calculus of approximations", Bulletin de l'Academie Polonaise de Sciences, 1956, Vol. 4, No. 5, pp. 253-257.

[21] I. M. Yaglom, Complex Numbers in Geometry, Academic Press, New York, 1968.

[22] L. A. Zadeh, "Fuzzy sets", Information and Control, 1965, Vol. 8, pp. 338-353. 